\title{
International Year of Light in Michigan: outdoor holography
}

John Nees, Cynthia Aku-Leh, Tim Jones, Anca Sala, Arlene Smith, et al.

John Nees, Cynthia Aku-Leh, Tim Jones, Anca L. Sala, Arlene Smith, Elizabeth F. C. Dreyer, "International Year of Light in Michigan: outdoor holography," Proc. SPIE 9946, Optics Education and Outreach IV, 99460B (27 September 2016); doi: 10.1117/12.2237426

Event: SPIE Optical Engineering + Applications, 2016, San Diego, California, United States 


\author{
International Year of Light in Michigan: outdoor holography \\ John Nees*a, Cynthia Aku-Leh ${ }^{\mathrm{b}}$, Tim Jones ${ }^{\mathrm{c}}$, Anca L. Sala ${ }^{\mathrm{d}}$, Arlene Smith ${ }^{\mathrm{e}}$, and Elizabeth F. C. \\ Dreyer $^{\mathrm{a}}$ \\ a Optics and Photonics Laboratory, University of Michigan, Ann Arbor MI, USA 48109; \\ ${ }^{\mathrm{b}}$ ISCIENCES, L.L.C., Ann Arbor MI, USA 48103; ${ }^{\mathrm{c}}$ ColorSpritz, Ann Arbor MI, USA 48103; \\ ${ }^{\mathrm{d}}$ College of Engineering, Baker College, Flint MI, USA 48507; ${ }^{\mathrm{e}}$ Avo Photonics, Inc., Horsham, PA, \\ USA 19044
}

\begin{abstract}
Outdoor holography is an activity created by the Michigan Light Project during the International Year of Light. Traditional holography is done in dark and quiet rooms. Using a kit from LitiHolo.com, we designed a way to make simple holograms outside in a noisy festival environment.
\end{abstract}

Keywords: Holography, IYL, International Year of Light, outdoor, outside, robust, outreach, K-12

\title{
1. INTRODUCTION
}

The 2015 International Year for Light led to the creation of many unique hands-on activities about optics and photonics phenomena and applications. Outdoor holography is an activity created by the Michigan Light Project during the International Year of Light (IYL). Traditional holography is done in dark and quiet rooms. The ability to make holography mobile and bring it to where the people are, namely outside, is essential to outreach. Using a commercial holography kit from LitiHolo (LitiHolo.com) as the base, we designed a way to make simple holograms outside in a noisy festival environment. Visitors learned about the basic concepts of holography such as the wave-nature of light and the combination of light waves to form interference. Visitors were able to make a hologram and take it home with them. We deployed the project at two large summer outdoor events -- Ann Arbor Summer Festival in Ann Arbor and Metro Youth Day at Belle Isle in Detroit -- with audiences from suburban and inner-city schools. The final glass-based hologram was always given to a guardian as a safety precaution. This paper details the learning objective and outcomes of the project as well as how to implement it in an outreach or festival event.

\subsection{International Year of Light}

As stated in the light2015.org homepage, "IYL2015 (was) a global initiative adopted by the United Nations to raise awareness of how optical technologies promote sustainable development and provide solutions to worldwide challenges in energy, education, agriculture, communications and health." An immense effort with 93 national nodes gathered around this initiative with the United Nations Educational, Scientific and Cultural Organization launching the event on 19 January 2015. Events celebrating the impact of light on the daily lives of people were held throughout the year to raise awareness of the nature of light and how it affects all aspects of life.

\subsection{Michigan Light Project}

In this context, the Michigan Light Project was established by a group of academics, industrialists, and artists to "demonstrate the amazing aspects of light and energy in society as well as promote awareness that the State of Michigan is a worldwide hub of light-related industry, culture, and art." Their mission was to use IYL as a catalyst to increase collaboration among artistic, industrial, and educational groups in addition to encouraging the local "economy of light" in Michigan. Like many sister organizations, the Michigan Light Project sorted through a plethora of ideas about identifying a common set of goals and about how to meet them with reasonably tangible resources. Drawing on a couple of campus-based clubs and people from the workplace, a couple of intense revisions of organizing principles were hammered before the IYL initiative began and a plan was set in motion to seek some funds both through the support of professional societies and through local companies. To flesh out the direction of the group's activities the range of

*nees@umich.edu

Optics Education and Outreach IV, edited by G. Groot Gregory, Proc. of SPIE Vol. 9946, 99460B

(C) 2016 SPIE · CCC code: $0277-786 \mathrm{X} / 16 / \$ 18 \cdot$ doi: $10.1117 / 12.2237426$ 
possible activities was narrowed down in order to allow for a wide range of impact and to increase the longevity of the message in the minds of people who might participate. This led our group toward a concentration on public festivals and

to activities that would allow hands-on participation with a 'take-home' component, and away from lecture and mediabased activities. The decision to join preexisting outdoor public events and festivals created an efficient means of contacting new people, but it also posed some challenges as to how to make a lasting impact. Thus, a few more sessions were needed to bring out ideas and to gauge their appeal. With many interesting ideas for microscopy, solar power, communications and the like were discussed and hashed out in crude prototypes, the group settled on a small subset that would provide a novel experience with a degree of safeguard against adverse weather and lighting conditions were chosen. Among these was the idea to make holograms that people could set up, expose and take home.

\subsection{Holograms - contagious, but not contagious}

In the current digital society, the allure of images may wane, but the intrigue a 3D image brings is curiously strong. From the movie image of Princess Leah leaving instructions for Luke to a 'pop-out' image of the day's activities Harry Potters copy of the Quibbler, the notion of people actually being able to use 3D imagery holds a consistent grip on the imagination. While holograms are not yet up to Hollywood standards, they do have a captivating ability to show an object in full 3D in 'thin air'. In another respect, holograms have found practical application to the point that nearly anyone reading this article could find on within reach on the surface of their credit card. With the combination of intrigue and utility is, nevertheless, almost unheard of for an average person to make a hologram. Fortunately, some degree of technological innovation has come into the science of holograms, and there is now a technology available that allows one to make their own hologram in a matter of minutes. Using a photopolymer to replace the more traditional and more cumbersome chemical development process, Liti Holographics (Litiholo.com) has developed a kit that bring the hidden art of holography home from the museum and the lab. We describe below how to use the kit in public settings such as festivals, family nights, to teach basic properties of light waves and how we make holograms in such settings.

\section{HOLOGRAPHY}

While Dennis Gabor's invention of holography predates that of the laser, it took the coherent properties of the laser and the move to photographic recording materials and off-axis designs to render images with remarkable 3D fidelity. Modern laser diodes are sufficiently stable to record holograms and polymeric recording media are now available that can reproduce holographic images without any chemical development procedures. In this section, we discuss the LitiHolo kit, the limitations we found in relation to our project, and the solutions that allowed us to use it in public places.

\subsection{The kit}

Liti Holographics' kit sells online for $\$ 99$ and it contains all the components needed to make a hologram. It provides a red laser diode powered at a safe level by a battery pack. A heat sink clip is included to help maintain laser diode stability. The exposure setup is constructed of laser-cut plastic with a vibration-damped base for the object and the film plate and a fitted piece to serve as a plate stabilizer. For convenience, the kit also has a blank test plate, a white alignment card, a black shutter card and a blue LED darkroom light. Its documentation includes a list of kit contents, an instruction manual, a basic concepts guide, and a set of important tips. Finally, the kit also includes a test object.

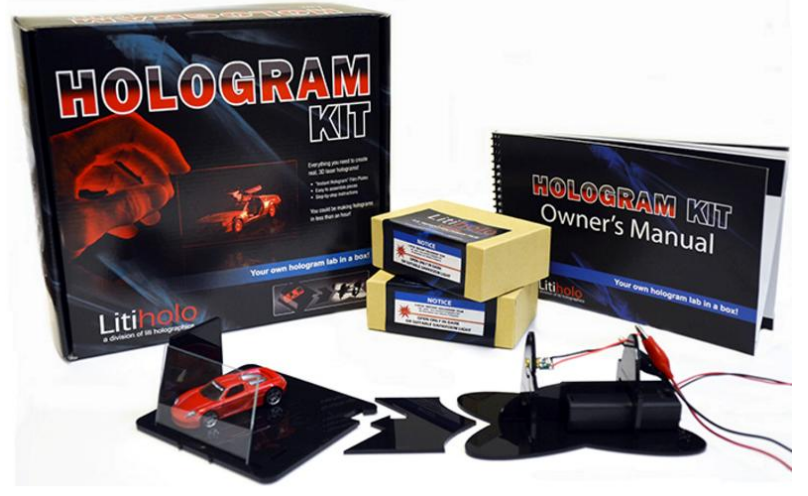

Fig. 1. Liti Holographics kit components as shown on the company's website ${ }^{1}$ 
Before proceeding to describe how we use this kit to make holograms in festival locations, it will be useful to remark on the advantages the kit provides over traditional holography, as many of these aspects also play as role in extending the use to the public arena. Following the owner's manual, all of the items shown in the foreground of Fig. 1 are assembled in a quiet, dark(ish), stable place. Then, with an unexposed film in the place of the glass plate, a four-minute exposure writes a transmission hologram of the test object. Following the exposure, the plate may be exposed to light for a while to exhaust the sensitizer and prevent further holographic recording. Whether this is done or not, the hologram may be viewed in the same configuration, but with the object removed. That's it!

Now we will proceed to note some advantages and some shortcomings of the system with respect to our interest in bringing this technique to public places. The first advantage is that there is no development procedure, only the requirement to stop writing the hologram. A photopolymer similar to that sold by Bayer (known for selling aspirin) is used in the recording plates. This more modern recording medium requires a significantly higher exposure fluence than traditional holographic plates, which also allows plates to be exposed to a considerable amount of light before use. On a sunny day, under the shade of a simple canopy tent, a novice can easily transfer a plate from its dark-box to its resting place in the holographic exposure configuration. In so doing, they can clearly see and touch the setup of the hologram before it is guarded by a dark enclosure for the exposure. On the other hand, the need for a long exposure also exacerbates the difficulties associated limits to coherence and stability.

The holographic photopolymer is quite capable of recording fringes generated in the configuration that the kit is designed for. The finest gratings written in the holographic material are a half wavelength in period, in this case $\sim 200 \mathrm{~nm}$. As is common to holographic procedures, it is necessary for the film and the object to be restricted to a significantly smaller range of motion during the entire exposure. It is also required that the laser remain substantially in the same wavelength over the writing time. Meeting these requirements avoids the production of multiple sets of holographic fringes that may wash out each other's diffraction efficiency. The kit uses a single beam technique to avoid many possible sources of motion. It also includes a clip that acts as a heat sink for the laser diode to reduce its wavelength drift due to thermal expansion or carrier density drift. It also reduces the diode's tendency to hop from one mode to another. Even with the recommended 5 minute warm-up time, holograms are still occasionally formed that display dark 'zebra stripes' every centimeter or so along the object. These bands are located at even steps along the object where the laser diode coherence is lost during the exposure, and loosely represent the coherence length of the light from the laser diode. Solutions do exist to extend the coherence length of laser diodes and to stabilize thermal drift, but are not presently available in the $\$ 1$ to $\$ 10$ range.

The single-beam design of the kit allows the left-hand portion of the laser beam directly expanding perpendicular to the plane of the diode junction to fall directly on the plate with and incidence angle near Brewster's angle, minimizing the reflection of the reference beam. The right-hand portion of the same beam illuminates the nearby object. This design minimizes variation of the physical path difference between the reference and object waves by eliminating mirrors and beam splitters that might move. It produces a beam free of the diffraction patterns of dust and scratches that might appear as a result of the use of such optics. Furthermore, by placing the object in contact with the holographic plate, there is at least one location where the reference and object waves are within a coherence length of each other. When an exposure passes without mode hopping in the diode a hologram is formed with a stripe-free depth of many centimeters.

As mentioned the image may be viewed in precisely the same configuration as the exposure, but without the object in place. Furthermore, the color of the illuminating laser may be changed and LEDs or white light may also be used. In the kit geometry discussed thus far, the laser-illuminated hologram is clearly visible, not only at the original illumination angle - and also at a range of angles nearby, thought with the introduction of some aberrations in the image. With LED illumination a range of chromatic blurring effects is apparent with a loss of fidelity that increases with distance from the hologram. In sunlight or with a white LED the image is clear only very near the plate. The result for the user is very satisfying if they possess the tools to 'replay' the image, but it is somewhat difficult to supply this arrangement to a novice holographer in a festival context. This is one limitation we addressed in a slight redesign of the kit for our project. 


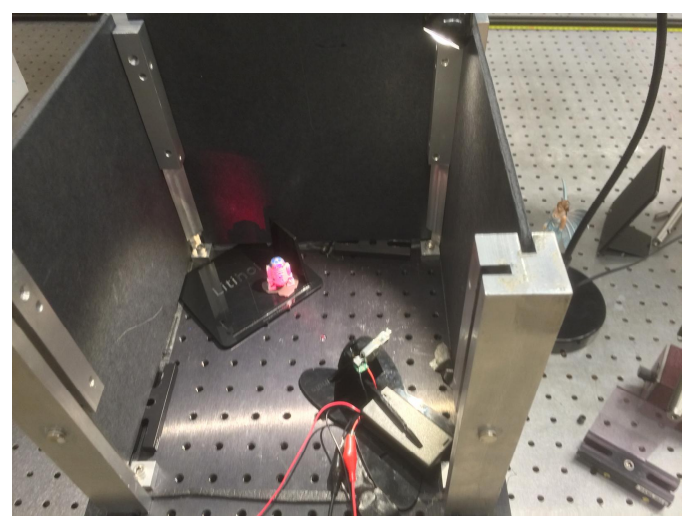

Fig. 2. Configuration used for one-minute exposure reflection hologram viewable with white light illumination—one side and cover removed.

The second element of the redesign concentrated on minimizing the exposure time to diminish to likelihood of motion or mode hopping during the exposure. As the laser power is constrained by law, this was addressed by leaving some of the active area of the hologram unexposed and by using the full beam as both reference and object wave in a reflectionhologram geometry. Beginning with the kit configuration, we rotated the laser $90^{\circ}$ to set the fast divergence angle in the vertical direction, we rotated the plate $90^{\circ}$ to portrait orientation, and we placed the laser on the opposite side of the plate from the object where the light would illuminate the object after passing through the plate. Finally, the distance from the laser to the holographic plate was reduced by a little less than a factor of two, leaving nominally half the plate unexposed.

The reflection hologram that is written in this geometry, while technically more susceptible to vibrations than the original transmission hologram, has one forth the exposure time. Also, the majority of its interference fringes are written with a period close to a half wavelength making its fringes more similar to those of a dielectric mirror and yielding better color selectivity. As a result, these fringes render better images in white light. Together, these features make the holograms more suitable as a hands-on activity with a take-home component.

We would like to note: it is a remarkable feat to place the capability to form holograms in such easy reach of novices as Liti Holographics has done; also, they now offer components to form reflection holograms in both in single-color and white-light configuration.

\subsection{A dark place}

A sunlit day in Michigan is perfect for festivals and public events, but direct sunlight is too strong for a 1-mW laser diode to compete with. To address this brightness issue and to address the possibility of rain, we carried out the holography activity within the shade of a canopy, ranging from a 3-meter square pop-up to an 8 meter by 16-meter rental tent with partial sides. Shading from direct sunlight and absorption of scattered light by surrounding grass or other groundcover has proved sufficient to allow the holographic plate to be handled by children and adults for the 20 to 40 seconds needed to place it in front of the object and to cover the setup with dark panels. We use a small plate $30-\mathrm{cm}$ square plate supported by $8 \mathrm{~cm}$ of closed-cell foam padding to support standards with vertical slots that accommodate black slide-in foam-core plates on four sides. We cover the setup with a soft black cover (a padded laptop case) that can be placed gently on the frame one exposure has commenced. These components provide protection from outside light and outside vibrations during exposure. To avoid bumping of the exposure chamber, we set it on a stand having a slightly larger cross-section. In short, the system reminds one of a magician's stand, though it is more rigid than a foldout stand. Naturally, we need to control traffic during exposures. We do this, in part, by using placards set up on a barrier table to explain the holographic process during this interval.

\subsection{A little time}

We have found that the intrigue of the hologram has been sufficient to command a relatively quiet atmosphere as long as the requirement for low vibrations, including sounds, is made known. All the same, the festival tent does not really support an atmosphere of extended patience, so we tend to keep a short line for participants by taking numbers and asking potential holographers to return after wondering for a specific time. Once a one-minute exposure begins, we ask for no talking close to the holography stand and turn away from it to discuss the important elements of the art. 


\subsection{Visual graphics}

Our learning objective is to introduce basic properties of light like superposition, interference, and diffraction, and at a more basic level, visual perspective. Making holograms forces one to adopt the ideas of visual perspective, where rays of light may have a simple character, as the reference wave does, or a complex nature as does the object wave. The concept that rays are transformed from the simple form to the complex form is a profound element that holography possess to a greater degree even than photography. We use four simplified drawings shown in Fig. 3 to convey the tools and three primary aspects of the hologram to a very broad range of the public, and we use more technical conversations to discuss the details of superposition, interference, and diffraction from the recording. The primary learning objective is simple.

The first graphic simply shows the elements of the system - the object, the viewer, the laser and its light, and the holographic film. The second graphic shows the ray configuration where the viewer sees an object by receiving the rays it scatters. In the third graphic, the exposure arrangement includes the holographic film. Moreover, in the final graphic, the film receives the laser light and delivers light scattered as though it came from the object, which is now removed. As the steps are reviewed we talk about the light directly from the laser recording a set of nanoscale mirror surfaces that are capable of redirecting the incoming wave into the complex object wave, but we focus on the concept that we perceive images based on the ray of light coming form objects. We convey that holograms use the wave properties of light to write a ray transformer.

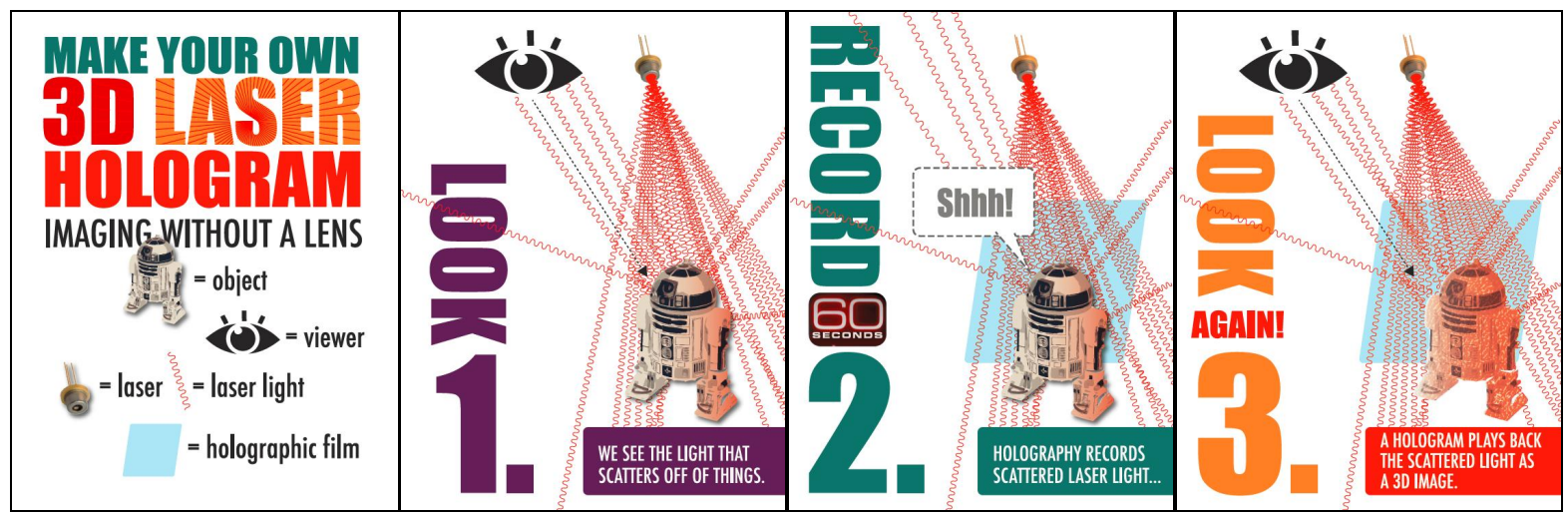

Fig. 3. Overview image describing materials needed for Hologram. 1. First step in Holography. 2. Second step for hologram - wait 60 seconds. 3. Final step for hologram - look at the hologram

\subsection{Teaching how to view hologram at home}

Clearly, the brief discussion of holography in the graphics above is not capable of fully teaching interference and diffraction in two minutes. We rely on the participant to discover further information on their own and we encourage them to show the hologram. This is the reason behind the move to a reflection hologram design that allows a broader range of illumination sources to be used to reconstruct the image. Fig. 4 shows a hologram illuminated by an LED desk lamp. The color shift from the original red writing color to yellow is due to the angles of illumination and viewing.

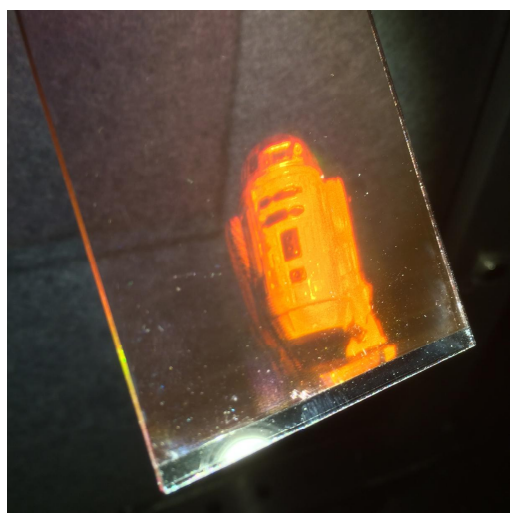

Fig. 4. Holographic image illuminated by white light 


\section{EVENTS}

Having prepared the reflection hologram setup as described above we took an afternoon to test it informally on the sidewalk of the University of Michigan's Central Campus. Picking a shaded area and taking more care not to allow too much exposure to sunlight, we invited passing college students to make holograms. Then, after several trials we took stock of our experience to formulate the graphics for the public events. In order to identify the holography activity we added a yard sign touting "MAKE YOUR OWN 3D LASER HOLOGRAM-one per kid while supplies last." Each of three primary events carried slightly different aspects that had an impact on the outcomes for participants, but the setup was adequate for all three.

First, we had scheduled a 'Kids Zone' activity (see Fig. 5) at the Ann Arbor Summer Festival, set in June of each year. This event lasts for about a month and hosts a children's activity tent on a majority of event days. Our booking included a variety of activities including microscopes, telescopes, 'pinky powered lights', and holography. This event proved that we could draw in visitors in spite of rain and that high humidity would not hinder the formation of good holograms. Still, the attendance was much lower than it might have been. Those who came either said they were there because they had read about the topics in the schedule or, a presenter in our group had specifically invited them. This afforded an intimate setting where one family would be able to make holograms for each child, and maybe an adult.

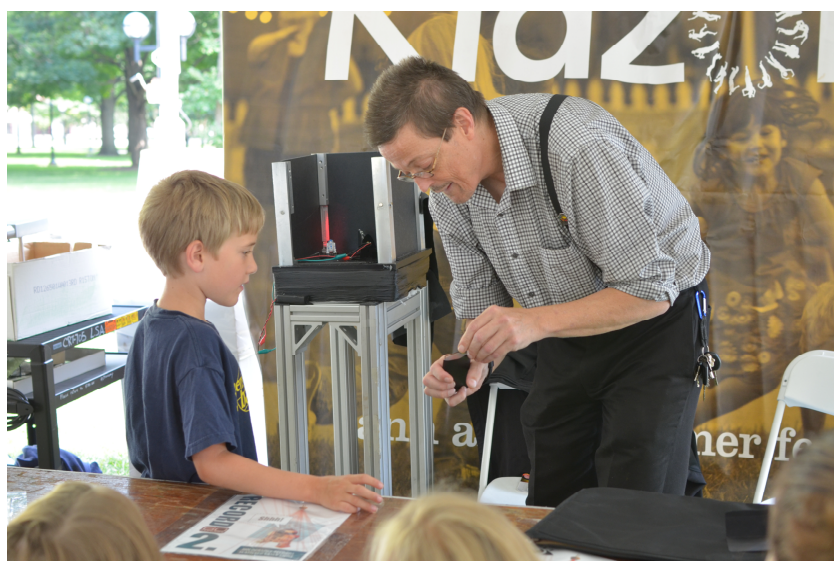

Fig. 5. Author J. Nees showing a hologram to a child during the Ann Arbor Summer Festival on 30 June 2015.

Our second event, Metro Youth Day, was set on a very sunny day in July on Detroit's Belle Isle. This was a high school reach out event with both recruiting and motivational aspects. Here, holography was set up in a tent with college promotional materials and giveaways (shown in Fig. 6), and we observed that the interest from the visitors varied evenly between the chaperones and their charges. There was a steady stream of participants over the full 5 hours of the event and we made 70 to 90 holograms. This rate indicates that the time per hologram is substantially longer than that required for setup and exposure. Therefore, the time spent in explanations is about twice the minimal time.

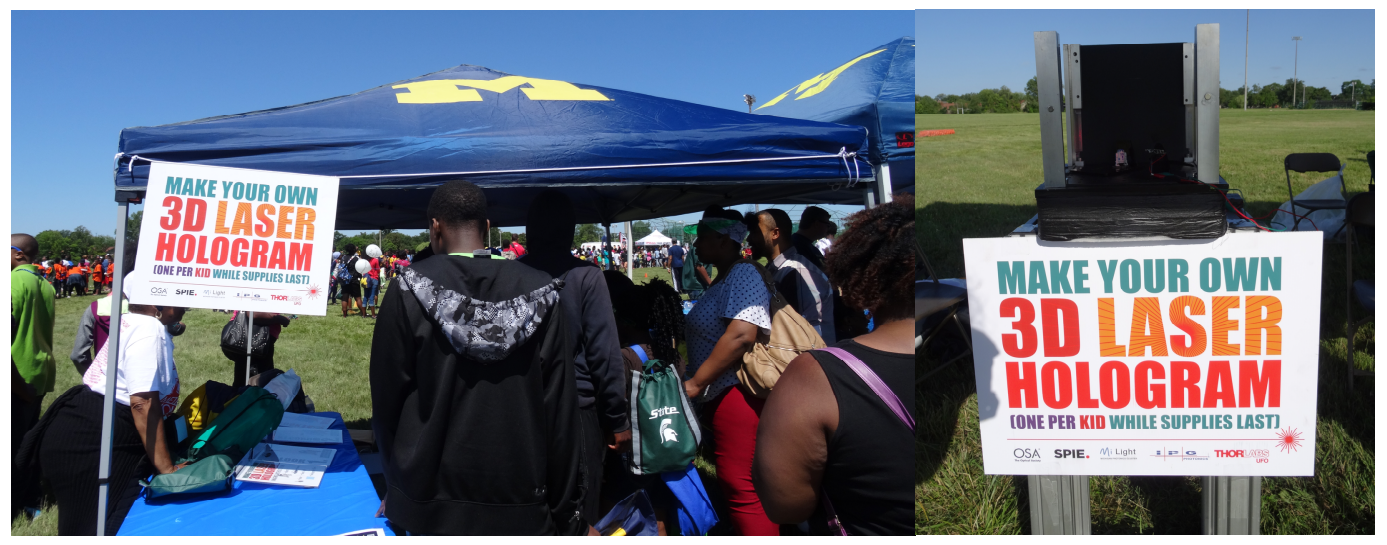

Fig. 6. (L) Tent at the Metro Youth Day in Belle Isle, Detroit, MI on 15 July 2015. (R) Close-up of holography apparatus. 
Our third event was indoors. We made holograms for the duration of the Electrical and Computer Engineering Family Night at the University of Michigan. The night included a laser light show and numerous hands-on exhibits of optical and electronic phenomena. Holography was positioned in front of a display of Emmitt Lieth's and Juris Upatnieks' more famous holograms, including the one shown in the 1966 Photography addition of Life magazine ${ }^{2}$. The audience was filled with knowledgeable enthusiasts on all manner of interference and diffraction. The presentation was a great way of connecting the older and younger generations with participants ranging in age from 6 to 80 .

Table 1. Summary of IYL Events with Outdoor Holography.

\begin{tabular}{|c|c|c|c|}
\hline Event Name & Location & Date & Audience Estimate \\
\hline Ann Arbor Summer Festival & Ann Arbor, MI & 30 Jun 2015 & 200 children + parents \\
\hline Metro Youth Day & Belle Isle, Detroit, MI & 15 Jul 2015 & $\begin{array}{c}300+\text { middle school and } \\
\text { high school students }\end{array}$ \\
\hline EECS Electrify - Light it Up & Univ. of Mich., Ann Arbor, MI & $20-25$ Jul 2015 & 30 high school students \\
\hline EECS Family Night & Univ. of Mich., Ann Arbor, MI & 25 Sep 2015 & 100 people, all ages \\
\hline
\end{tabular}

\section{RECOMMENDATIONS FOR IMPLEMENTATION}

After interacting with participants of all ages and backgrounds at four different events, we have compiled the following recommendations for implementation.

\subsection{Event Logistics}

For festival-like environments where there are many people and the potential for long lines, it is essential to have at least two volunteers. With two volunteers, one person can be in charge of making the holograms while the other is engaging with people in the line. Since the holograms take about two minutes per exposure, it is recommended to have siblings or groups share one hologram rather than each person make their own. For safety, the completed hologram is placed in a black plastic trading card sleeve and then given to an adult. The black backing on the sleeve allows for easier viewing. These can be purchased online or at a local trading card or game store.

Additionally, we recommend that organizers bring many batteries to any event. The lasers run best using batteries since batteries have less electrical noise than standard wall outlets. However, the batteries can die quickly if kept on for hours. We recommend using rechargeable batteries as well to offset the environmental impact.

\subsection{Alternative to LitiHolo kit}

Although we used the kit from LitiHolo, this activity could be done with a different laser source and holographic film. The laser must have a diverging beam capable of covering the desired target object. We recommend using holographic film that can be developed using UV light or sunlight. Chemical exposures can be messy and dangerous for working with large crowds or young children.

\section{CONCLUSIONS}

We have modified a commercial photopolymer-based holography kit for use in public festivals and gatherings. The changes improved at-home viewing to allow white light illumination and diminished recording time fourfold. In a wide variety of situations from rain to shine, the modified kit performed admirably. During the International Year of Light, we made hundreds of holograms and conveyed knowledge about the capability of a hologram to act as a light wave transformer to produce a 3D image of the recorded object. Apart from IYL activities, we have also used the holography kits in a junior high summer camp and as an optics laboratory tool to initiate visiting undergraduate students into a small subset of optical practices. In all these circumstances the results of participating in setting up and exposing holograms has been a heightened interest and awareness of optical processes. 


\section{ACKNOWLEDGEMENTS}

The authors would like to acknowledge the International Society for Optics and Photonics (SPIE), the Optical Society (OSA), Mi-Light, IPG-Photonics, and Thorlabs-UFO for their financial support of these activities. They would also like to acknowledge all of the volunteers that made these events possible, especially those from the Optical Society at the University of Michigan (OSUM), the Ann Arbor OSA, the Student Astronomical Society (SAS) at the University of Michigan, and Baker College of Flint. Furthermore, thank you to all of the festivals that let us participate and all of the people who stopped by our tables and enjoyed the wonder of light.

\section{REFERENCES}

[1] Photograph used with permission of Paul Christie of Liti Holographics, Newport News VA, http://www/http://litiholo.com/hologram_kits.html

[2] Life, 61 (26), 23 Dec 1966: 104-19 (1966). 